\title{
When the unusual is the normal: making non-standard structural studies routine.
}

\author{
A Wheaton ${ }^{1}$, I Guzei ${ }^{2}$ \\ ${ }^{1}$ University of Wisconsin at Madison Department of Chemistry, Madison, WI, ${ }^{2}$ Chemistry, UW \\ Madison \\ amwheaton@wisc.edu
}

We present several problematic small-molecule structures that required non-routine handling of data collection, processing and/or structural refinement. The structure of an octahedral $\mathrm{Ni}$ (II) complex could not be properly refined until the crystal was identified as a pseudo-merohedral twin. A highly absorbing complex [C[sub]6[/sub]H[sub]5[/sub]CH[sub]2[/sub]CH[sub]2[/sub]NH[sub]3[/sub]][sub]2[/sub][CH[sub]3[/sub]NH[sub]3[ $/$ sub]][Pb[sub]2[/sub][[sub]7[/sub]] $(\mu \mathrm{Mo}=25.54 \mathrm{~mm}[\mathrm{sup}]-1[/ \mathrm{sup}])$ required a careful choice of crystal, radiation source, and subsequent positional disorder modelling. A sample of an organic salt contained non-merohedrally twinned crystals only; upon exploring multiple absorption correction choices with TWINABS, the structural model necessitated use of the Platon SQUEEZE protocol for modeling of disordered solvent electron density. A Co(II) complex required lowering the crystallographic symmetry from space group $[\mathrm{i}] \mathrm{C} / \mathrm{i}] 2 /[\mathrm{i}] \mathrm{c}[/ \mathrm{i}]$ to $[\mathrm{i}] \mathrm{C} / \mathrm{i}][\mathrm{i}] \mathrm{c}[/ \mathrm{i}]$, identifying solvent of crystallization, and then finally establishing the chemical identity of the metal center. Finally, a chiral [i] $\mathrm{P}[/ \mathrm{i}] 1$ organic salt whose crystals decomposed due to radiation damage during the X-ray measurement required two data collections to achieve a data set that had a sufficient data completeness for the subsequent structural refinement process.

Acta Cryst. (2020). A76, a15 